\title{
Síndrome de deleção 22q11.2 e cardiopatias congênitas
}

\author{
22q11.2 deletion syndrome and congenital heart defects
}

Rafael Fabiano M. Rosa', Paulo Ricardo G. Zen², Carla Graziadio³, Giorgio Adriano Paskulin ${ }^{4}$

\section{RESUMO}

Objetivo: Revisar as características clínicas, etiológicas e diagnósticas da síndrome de deleção 22q11 e sua associação com as cardiopatias congênitas.

Fontes dos dados: Foram pesquisados artigos científicos presentes nos portais Medline, Lilacs e SciELO, utilizando-se descritores específicos como "22q11", "DiGeorge syndrome", "velocardiofacial syndrome", "congenital heart defects" e "cardiovascular malformations". O período adotado para a revisão foi de 1980 a 2009.

Síntese dos dados: As malformações cardíacas são os defeitos congênitos observados mais frequentemente ao nascimento e representam um problema importante de Saúde Pública. Dentre suas principais causas conhecidas destaca-se a síndrome de deleção 22q11, também chamada de síndrome de DiGeorge, síndrome velocardiofacial e CATCH22. Trata-se de uma doença autossômica dominante caracterizada por um fenótipo altamente variável, o que dificulta em muito seu reconhecimento clínico. Além disso, a maior parte dos pacientes apresenta uma microdeleção identificada principalmente por técnicas de citogenética molecular, como a hibridização in situ fluorescente, pouco disponíveis em nosso meio. De forma similar a outras síndromes, a síndrome de deleção 22q11 associa-se a certos defeitos cardíacos específicos, no caso os do tipo conotruncal. Apesar disso, não há ainda na literatura um consenso sobre quais os pacientes com cardiopatia congênita que deveriam ser investigados para a síndrome de deleção 22q11.

Instituição: Universidade Federal de Ciências da Saúde de Porto Alegre (UFCSPA) e Complexo Hospitalar Santa Casa de Porto Alegre (CHSCPA), Porto Alegre, RS, Brasil

'Doutorando do Programa de Pós-Graduação em Patologia da UFCSPA; Geneticista Clínico do Hospital Materno Infantil Presidente Vargas (HMIPV), Porto Alegre, RS, Brasil

2Doutor pelo Programa de Pós-Graduação em Patologia da UFCSPA; Geneticista Clínico da UFCSPA e do CHSCPA, Porto Alegre, RS, Brasil ${ }^{3}$ Doutoranda do Programa de Pós-Graduação em Patologia da UFCSPA; Geneticista Clínica da UFCSPA e CHSCPA, Porto Alegre, RS, Brasil ${ }^{4}$ Doutor pelo Programa de Pós-Graduação em Genética e Biologia Molecular da Universidade Federal do Rio Grande do Sul (UFRGS); Professor da Disciplina de Genética Clínica e do Programa de Pós-Graduação em Patologia da UFCSPA, Porto Alegre, RS, Brasil
Conclusões: Cardiologistas e cirurgiões cardíacos, especialmente pediátricos, devem estar cientes das peculiaridades e dos cuidados dispensados à síndrome de deleção 22q11. Os indivíduos com a síndrome apresentam comumente alterações envolvendo vários sistemas, o que pode levar a dificuldades e a complicações durante seu manejo clínico e cirúrgico.

Palavras-chave: síndrome de DiGeorge; hibridização in situ fluorescente; cardiopatias congênitas; cromossomos humanos, par 22.

\section{ABSTRACT}

Objective: To review clinical, etiological and diagnostic characteristics of the 22q11 deletion syndrome and its association with congenital heart defects.

Data sources: Medline, Lilacs and SciELO databases were searched from 1980 to 2009 using specific descriptors as "22q11", "DiGeorge syndrome", "velocardiofacial syndrome", "congenital heart defects" and "cardiovascular malformations".

Data synthesis: Heart malformations are the most frequent congenital defects at birth and represent an important Public Health problem. The 22q11 deletion syndrome, also called DiGeorge syndrome, velocardiofacial syndrome and CATCH22, stands out as one of the main known causes of congenital heart defects. This is an autosomal dominant genetic disease characterized by a highly variable phenotype, which renders its difficult clinical identification. In addi-

\section{Endereço para correspondência: \\ Giorgio Adriano Paskulin \\ Rua Sarmento Leite, 245, sala 403 \\ CEP 90050-170 - Porto Alegre/RS \\ E-mail: paskulin@ufcspa.edu.br}

Fonte financiadora: Coordenação de Aperfeiçoamento de Pessoal de Nível Superior (CAPES); Programa de Pós-Graduação em Patologia da UFCSPA; University of Colorado, Aurora, Colorado, Estados Unidos

Conflito de interesse: nada a declarar

Recebido em: 18/12/2009

Aprovado em: 11/6/2010 
tion, the majority of the patients present a microdeletion identified mainly by molecular cytogenetic techniques as fluorescent in situ hybridization, which are rarely available in Brazil. Similarly to other syndromes, 22q11 deletion syndrome is associated to some specific heart defects, especially conotruncal. It is still not clear which patients with congenital heart defect should be screened for 22q11 deletion syndrome.

Conclusions: Cardiologists and cardiac surgeons, particularly the pediatric ones, must be aware about the features and health care related to $22 \mathrm{q} 11$ deletion syndrome. Subjects with the syndrome very often present abnormalities of multiple systems, that could result in difficulties and complications during their clinical and surgical course.

Key-words: DiGeorge syndrome; in situ hybridization, fluorescence; heart defects, congenital; human chromosome, pair 22.

\section{Introdução}

A doença cardíaca congênita é considerada a mais comum de todas as malformações, correspondendo a mais de $40 \%$ de todos os defeitos identificados ao nascimento ${ }^{(1,2)}$. Tratase de um grupo heterogêneo de lesões com consequências hemodinâmicas variáveis e, assim, com diferentes necessidades de seguimento e intervenção ${ }^{(1)}$. As comunicações interventriculares (CIV), na forma isolada, são, de longe, a alteração anatômica congênita mais comum do coração e estão presentes em $33 \%$ de todas as crianças afetadas ${ }^{(3)}$. Os defeitos cardíacos conotruncais caracterizam-se por alterações nas vias de saída do coração e correspondem a cerca de 20 a $50 \%$ das cardiopatias congênitas encontradas em recém-nascidos. Fazem parte desse grupo a tetralogia de Fallot (TOF), a interrupção do arco aórtico (IAA), o truncus arteriosus (TA), a comunicação interventricular com atresia pulmonar (CIV/ AP), a transposição das grandes artérias (TGA) e a dupla via de saída do ventrículo direito (DVSVD) ${ }^{(4)}$.

A incidência das cardiopatias congênitas varia entre quatro a 14 por 1.000 nascidos vivos, sendo que vários fatores podem influenciar em sua avaliação epidemiológica, como a ocorrência de defeitos letais que impossibilitam o nascimento vivo do concepto, o aborto terapêutico por doenças cardíacas complexas e a idade dos pacientes ${ }^{(3)}$. A principal ferramenta utilizada no diagnóstico e a indicação cirúrgica das doenças cardíacas congênitas é a ecocardiografia bidimensional com Doppler a cores, que se tornou mais acessível a partir de
$1988^{(1)}$. Entretanto, a modalidade diagnóstica definitiva, que provê dados hemodinâmicos, anatômicos e eletrofisiológicos críticos para o cuidado com o paciente, continua sendo o cateterismo cardíaco. Embora a cardiologia fetal tenha adicionado ao nosso conhecimento a doença cardíaca congênita na vida fetal, sua principal função é identificar malformações que necessitam de abordagem imediata após o nascimento ou que estão associadas a um prognóstico reservado. É sabido que a identificação desses pacientes ainda no período prénatal é capaz de reduzir substancialmente suas chances de mortalidade e de morbidade perinatais, pois possibilita que o nascimento em centros com condições mínimas para sua avaliação e seu tratamento ${ }^{(5)}$.

Os defeitos cardíacos congênitos graves e moderadamente graves, que irão necessitar de cuidados cardiológicos avançados, são encontrados em cerca de três a quatro em cada 1.000 nascidos vivos ${ }^{(2,3)}$ e representam uma causa importante tanto de admissão como de mortalidade em pacientes atendidos em unidades pediátricas de tratamento intensivo ${ }^{(6)}$.

Assim, apesar da redução progressiva da mortalidade ocorrida nas últimas décadas, as cardiopatias congênitas ainda representam um problema maior de Saúde Pública no mundo. Elas constituem a principal causa de óbito no primeiro ano de vida, sendo que suas formas mais graves, como a TOF, a TGA e o TA, correspondem a $20 \%$ dos óbitos neonatais. Tal situação se agrava em locais onde há falta de cuidados médicos e cirúrgicos precoces, pois cerca de $50 \%$ dos portadores de cardiopatia congênita precisam ser operados no primeiro ano de vida ${ }^{(2)}$.

O baixo peso ao nascer, a prematuridade, a estadia prolongada em unidade de tratamento intensivo, a doença cardíaca complexa e o retardo do crescimento intrauterino são fatores associados à mortalidade mais elevada em crianças com cardiopatia congênita. Pacientes com alterações extracardíacas e/ou associadas a síndromes genéticas também apresentam um risco maior de morbidade e mortalidade, mesmo no momento da cirurgia cardíaca. Esses indivíduos podem também requerer intervenção de natureza cirúrgica ou clínica independentemente do problema cardíaco ${ }^{(7)}$.

\section{Etiologia das cardiopatias congênitas}

A etiologia das malformações cardíacas é pouco compreendida ${ }^{(8)}$. Somente em 10 a $25 \%$ dos casos é possível fazer uma associação com causas conhecidas, como doenças genéticas de origem gênica e multifatorial, doenças maternas e teratógenos conhecidos ${ }^{(9)}$. As anormalidades cromossômicas 
são mais frequentes em pacientes com malformações cardíacas congênitas do que na população em geral. Antes da década de 1990, com o uso de técnicas de cariotipagem convencionais, elas eram identificadas em 8 a $13 \%$ dos pacientes. A síndrome de Down é considerada ainda hoje a alteração cromossômica mais observada em indivíduos com cardiopatia congênita, sendo verificada em 4 a $10 \%$ desses pacientes ${ }^{(10)}$.

Posteriormente, com o início da análise pela hibridização in situ fluorescente (FISH), técnica que integra o uso da citogenética clássica com a genética molecular, uma série de anormalidades começou a ser descrita e outras foram mais bem caracterizadas ${ }^{(11)}$. Vários relatos com diferentes abordagens utilizando essa técnica têm sido publicados, incluindo sua aplicação na detecção de microdeleções cromossômicas em doenças genéticas específicas, como a síndrome de deleção 22q11.2 (OMIM \#192430 e \#188400) $)^{(12)}$. Identificada primeiramente no início da década de 1990, essa síndrome é considerada hoje uma das doenças genéticas mais frequentes em pacientes com cardiopatia congênita ${ }^{(13)}$.

\section{Síndrome de deleção 22q11.2}

A síndrome de deleção 22q11.2 (SD22q11), também conhecida como síndrome de DiGeorge, síndrome velocardiofacial, síndrome de Sedlačková, síndrome de Cayler, síndrome de Shprintzen e CATCH22 (Conotruncal heart defect, Abnormal face, T-cell deficiency, Clefting, e Hypocalcemia, decorrentes de uma anormalidade no cromossomo 22) é uma síndrome comum, mas pouco diagnosticada. Apresenta uma prevalência estimada de um para cada 2.000 a 6.000 nascimentos, o que faz dela uma das doenças genéticas mais frequentes no homem. Caracteriza-se clinicamente por um fenótipo altamente variável, sendo que, até o momento, mais de 180 achados clínicos, incluindo anormalidades físicas e comportamentais, já foram descritos na síndrome. Contudo, nenhum deles é obrigatório, o que acaba dificultando sua identificação. Apesar disso, existem alguns achados considerados mais característicos e que deveriam levar a um alto índice de suspeição, como a presença de defeitos cardíacos congênitos do tipo conotruncal ${ }^{(13,14)}$ (Quadro 1).

Atualmente, sabe-se que o braço longo do cromossomo 22 apresenta um arranjo não usual, com regiões de repetições de baixo número de cópias (low-copy repeats, LCRs) essencialmente idênticas, que predispõem a região q11.2 à deleção. A maior parte dos pacientes com a SD22q11 apresenta uma deleção de cerca de 3 milhões de pares de base $(\mathrm{Mb})$ na região 11.2 do braço longo do cromossomo 22 , sendo que o sequenciamento desse segmento cromossômico revelou a presença de aproximadamente 30 genes. Entre eles, está o TBX1 (T-BOX 1), expresso nas células derivadas da crista neural e que parece ter relação com os achados clínicos observados na síndrome, em especial com os defeitos cardíacos congênitos. As células da crista neural apresentam grande importância no desenvolvimento do septo conotruncal do coração, sendo que as malformações cardíacas envolvendo essa região são frequentes em pacientes com a SD22q11. Contudo, a grande variabilidade clínica observada na síndrome, mesmo entre indivíduos de uma mesma família, tem feito com que as associações genótipo-fenótipo não sejam possíveis ${ }^{(13,15)}$.

Na maior parte das vezes, a deleção 22q11.2 ocorre devido a mutações novas (de novo); porém, em 8 a $28 \%$ dos casos, ela é herdada e segrega-se nas famílias com um padrão de herança autossômico dominante, ou seja, com um risco de $50 \%$ dos indivíduos afetados transmitirem a deleção a seus filhos. Ambos os pais de um paciente com a SD22q11 devem ser testados para a deleção, pois podem apresentar uma expressão leve da síndrome. Essa informação é fundamental para o aconselhamento genético adequado do indivíduo acometido e de sua família ${ }^{(13,16)}$.

O diagnóstico da SD22q11 é usualmente confirmado da seguinte forma: primeiro realiza-se o estudo citogenético por meio do cariótipo de alta resolução por bandas GTG. Este é capaz de identificar até $15 \%$ dos pacientes com deleção em 22q11.2. Caso essa primeira avaliação seja normal, o próximo

Quadro 1. Achados frequentemente observados em pacientes com a SD22q11, divididos de acordo com a faixa etária

\begin{tabular}{|lcc|}
\hline Período neonatal & Infância & Idade adulta \\
\hline Hipocalcemia & Anormalidades palatinas & Dismorfias faciais \\
Cardiopatia congênita & Dificuldade de fala & Transtornos comportamentais \\
Imunodeficiência & Dificuldade de aprendizagem & Dificuldade de fala \\
Anomalias renais & RDNPM & Dificuldade de aprendizagem \\
Agenesia/hipoplasia de timo & Baixa estatura & \\
\hline
\end{tabular}

Baseado em Rosa et al (13). RDNPM: retardo no desenvolvimento neuropsicomotor. 
passo é realizar a pesquisa de microdeleção 22q11.2 pela técnica de FISH, pois a maioria dos pacientes possui uma deleção muito pequena (microdeleção), detectável somente por esse exame. Assim, o uso combinado do cariótipo de alta resolução e da técnica de FISH é capaz de identificar mais de 90 a $95 \%$ dos casos de SD22q11 $1^{(13)}$.

Deve-se ter em mente também que a falta de identificação da deleção por essas técnicas não exclui a presença de anormalidades na região 22q11.2, pois alguns pacientes $(<5 \%)$ podem apresentar alterações menores dentro de genes específicos, detectadas somente por técnicas moleculares, como o sequenciamento. Apesar desses métodos serem utilizados no momento quase que exclusivamente em nível de pesquisa, alguns deles, como a reação em cadeia da polimerase (PCR), mostram-se bastante promissores, devido, principalmente, aos seus menores custos e ao tempo de resposta ${ }^{(13,17)}$.

\section{SD22q11 e cardiopatias congênitas}

A SD22q11 é considerada atualmente uma das anormalidades cromossômicas mais frequentemente observadas em associação com defeitos cardíacos congênitos, ficando atrás apenas da síndrome de Down. Contudo, sua frequência entre esses pacientes é variável, oscilando entre 1 a 19\%, o que pode, em parte, ser explicado pela diversidade tanto das amostras analisadas como dos métodos empregados nos estudos $^{(18-27)}$. No único trabalho realizado em nosso meio, Rosa et al, avaliando pacientes hospitalizados em uma unidade cardiológica de tratamento intensivo de um hospital pediátrico, encontraram $2 \%$ de pacientes com SD22q11, resultado similar ao de estudos realizados em outros países envolvendo pacientes portadores de defeitos cardíacos maiores $^{(26)}$ (Tabela 1).

Indivíduos com as síndromes de Down, Noonan e HoltOram apresentam, de forma geral, tipos distintos de defeitos cardíacos que muitas vezes as caracterizam. Contribuições recentes indicam que isso parece acontecer também em pacientes com a SD22q11 $1^{(22)}$. Os tipos anatômicos de defeitos cardíacos mais associados à síndrome pertencem ao subgrupo dos defeitos conotruncais, sendo que a SD22q11 é observada em 4 a $48 \%$ desses pacientes ${ }^{(28-42)}$ (Tabela 2). Malformações cardíacas frequentemente associadas à deleção incluem a TOF (observada em 17 a $63 \%$ dos pacientes com a SD22q11), a IAA (em especial do tipo B, cuja interrupção se localiza entre as artérias subclávia e a carótida esquerdas; 5 a 23\%), a CIV/ $\operatorname{AP}(17$ a $27 \%)$ e o TA $(1 \text { a } 11 \%)^{(22,24,43)}$. A CIV, usualmente perimembranosa ou subarterial, ocorre em 10 a $50 \%$ dos pacientes com a SD22q11 $1^{(44)}$.

Por outro lado, a SD22q11 é descrita em 48 a $60 \%$ dos pacientes com IAA (principalmente do tipo B) ${ }^{(45,46)}, 31$ a

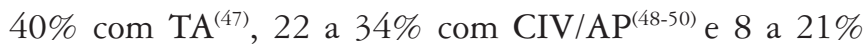
com $\operatorname{TOF}^{(26,50-53)}$. As anomalias do arco aórtico são também significativamente mais frequentes em pacientes com a SD22q11, sendo que a ocorrência isolada desses defeitos está associada à síndrome em cerca de $24 \%$ dos $\operatorname{casos}^{(54)}$. Embora a DVSVD e a TGA sejam defeitos cardíacos conotruncais, deleções 22q11.2 têm sido pouco descritas nesses pacientes ${ }^{(22,43)}$. Apesar de também menos frequentes, há descrições da SD22q11 em indivíduos com outros defeitos cardíacos, como a comunicação interatrial e o defeito de septo

Tabela 1 - Dados de estudos que avaliaram a frequência da SD22q11 em amostras não selecionadas de pacientes com cardiopatia congênita

\begin{tabular}{|c|c|c|c|c|c|c|c|c|c|}
\hline \multirow{2}{*}{ Trabalhos } & \multirow{2}{*}{$\begin{array}{l}\text { Idade } \\
\text { (anos) }\end{array}$} & \multirow{2}{*}{$\mathbf{N}$} & \multicolumn{5}{|c|}{ Cardiopatias congênitas* } & \multirow{2}{*}{$\begin{array}{l}\text { Cariótipo } \\
\text { anormal }\end{array}$} & \multirow{2}{*}{$\begin{array}{c}\text { SD22q11 } \\
\text { (\%) }\end{array}$} \\
\hline & & & IAA & TA & TOF & CIVIAP & Outras & & \\
\hline Giray et al(23) & ND & 36 & - & - & $11(2)$ & $5(3)$ & $20(2)$ & ND & $7(19,4)$ \\
\hline Schellberg et a/25) & ND & 312 & ND & ND & ND & ND & ND & $30^{\dagger}$ & $43(13,8)$ \\
\hline Marino et $\mathrm{a} l^{(22)}$ & $0-15$ & 931 & ND (8) & ND (10) & ND (23) & ND (24) & ND (22) & ND & $88(9,5)$ \\
\hline Fokstuen et a/(18) & $0-16$ & 110 & $2(1)$ & $5(2)$ & $16(1)$ & $8(3)$ & $79(2)$ & 0 & $9(8,2)$ \\
\hline Borgmann et al(20) & $0-27$ & 182 & $1(1)$ & $5(1)$ & $25(6)$ & 1 & $150(2)$ & $\mathrm{ND}^{+}$ & $10(5,5)$ \\
\hline Goodship et a/(19) & ND & 170 & $4(2)$ & ND & ND & $10(2)$ & ND (2) & 21 & $5(2,9)$ \\
\hline Hu et $\mathrm{al}^{(27)}$ & $0-63$ & 241 & 1 & 2 & 36 & - & 202 & 4 & $7(2,9)$ \\
\hline Rosa et a/(26) & $0-13$ & 198 & - & 3 & $20(2)$ & 5 & $(2)$ & 29 & $4(2)$ \\
\hline Botto et $a /^{(24)}$ & ND & 2396 & $17(8)$ & $21(4)$ & $125(9)$ & ND (6) & ND (9) & ND & $35(1,5)$ \\
\hline Yong et $a^{(21)}$ & ND & 87 & ND & ND & 15 & ND (1) & ND & $\mathrm{ND}^{+}$ & $1(1,2)$ \\
\hline
\end{tabular}

*Os números entre parênteses correspondem ao número de casos com a SD22q11 em pacientes com o respectivo defeito cardíaco. ${ }^{\dagger}$ Estudos com exclusão, em suas amostras, de pacientes com anormalidades cromossômicas numéricas, como trissomias dos cromossomos 13, 18 e 21. N: número de pacientes testados para microdeleção 22q11.2 pela técnica de FISH ou MLPA; IAA: interrupção de arco aórtico; TA: truncus arteriosus; TOF: tetralogia de Fallot; CIVIAP: comunicação interventricular com atresia pulmonar; ND, não descrito. 
atrioventricular $^{(22,24,26)}$. Além disso, pacientes com a SD22q11 podem, frequentemente, apresentar anomalias adicionais em quatro áreas do sistema cardiovascular:

1. Arco aórtico: pode estar localizado à direita, ser cervical e duplo, e a artéria subclávia originária do mesmo pode ser aberrante.

2. Artérias pulmonares: podem apresentar descontinuidade, hipoplasia difusa, estenose, defeito de arborização e artérias colaterais aortopulmonares maiores.

3. Septo infundibular: pode ser mal-alinhado, hipoplásico ou ausente. Este é virtualmente sempre envolvido, pois ele é mal-alinhado e/ou ausente em indivíduos com TOF, CIV/AP e IAA, e sempre ausente naqueles com TA.

4. Válvulas semilunares: podem ser bicúspides, muito displásicas, insuficientes ou estenóticas ${ }^{(22,34,54,55)}$.

A frequência geral de malformações cardíacas em pacientes com a SD22q11 varia de 49 a $87 \%{ }^{(43,56)}$. Contudo, estudos com indivíduos diagnosticados após os seis meses de idade ${ }^{(54)}$ e na idade adulta ${ }^{(57,58)}$ indicam índices muito menores desses defeitos (38\% e $25-30 \%$, respectivamente). Cohen et al encontraram uma diferença significante entre a frequência dessas malformações em crianças e pacientes adultos ${ }^{(57)}$, sendo que esse achado parece ter relação com o fato de que o óbito nos pacientes com a SD22q11 ocorre principalmente dos primeiros meses de vida em decorrência de complicações relacionadas às cardiopatias congênitas ${ }^{(43)}$. A mortalidade parece se associar tanto à gravidade das lesões cardíacas como à presença de anormalidades extracardíacas ${ }^{(59)}$. Os trabalhos que avaliaram pacientes com cardiopatia do tipo conotruncal em diferentes idades têm também verificado que a frequência da SD22q11 é inferior em pacientes adultos ${ }^{(39)}$ do que em menores de um mês de idade ${ }^{(35)}(5,8$ e $48 \%$, respectivamente), sugerindo uma alta mortalidade precoce. Shooner $e t a l$, por sua vez, acreditam que a prevalência de cardiopatias congênitas na SD22q11 seja, de forma geral, superestimada, devido à seleção de casos esporádicos de pacientes com defeitos cardíacos congênitos ${ }^{(60)}$.

Os estudos pré-natais descrevem frequências mais elevadas da SD22q11 em portadores de defeitos cardíacos do que as observadas em trabalhos pós-natais. Possivelmente, essa diferença ocorra devido ao óbito perinatal de fetos/neonatos com malformações cardíacas complexas ${ }^{(61)}$. Nessa mesma linha, Rosa et al propuseram, em seu trabalho, que o baixo índice de detecção pré-natal encontrado na região estudada (somente 12\%) possa ter influenciado a frequência verificada da SD22q11. É possível que pacientes com a SD22q11 portadores de cardiopatia congênita grave tenham falecido antes de ter acesso a um hospital terciário, levando à subestimativa de sua frequência. Cabe salientar que, no Brasil, ao contrário de alguns países desenvolvidos, o aborto seletivo para malformações é proibido por lei ${ }^{(26)}$. Por outro lado, uma

Tabela 2 - Frequência da SD22q11 em amostras de pacientes portadores de cardiopatia conotruncal (em ordem decrescente de frequência da síndrome)

\begin{tabular}{|c|c|c|c|c|c|c|c|c|}
\hline \multirow{2}{*}{ Trabalhos } & \multirow{2}{*}{ Idade } & \multirow{2}{*}{ N } & \multicolumn{5}{|c|}{ Cardiopatia conotruncal* } & \multirow{2}{*}{$\begin{array}{l}\text { SD22q11 } \\
\text { (\%) }\end{array}$} \\
\hline & & & IAA & TA & TOF & CIVIAP & Outros & \\
\hline Iserin et $a^{(35)}$ & RN & 104 & $18(16)$ & $17(7)$ & $60(21)$ & - & $9(6)$ & $50(48)$ \\
\hline Derbent et $a /(37)$ & $1 m-9 a$ & 30 & - & 3 & $7(4)$ & ND (1) & ND (4) & $9(30)$ \\
\hline Goldmuntz et $a /^{(28)}$ & ND & 17 & 3 & $4(3)$ & $10(2)$ & - & - & $5(29)$ \\
\hline Goldmuntz et a/(34) & ND & 251 & $24(12)$ & $29(10)$ & $126(20)$ & - & $72(3)$ & $45(18)$ \\
\hline Webber et $a /^{(31)}$ & $1 d-4 m$ & 46 & $4(2)$ & 3 & $23(1)$ & $11(3)$ & $5(2)$ & $8(17)$ \\
\hline Worthington et $\mathrm{a}^{/(33)}$ & ND & 90 & $6(3)$ & 10 & $48(6)$ & $17(4)$ & $9(2)$ & $15(17)$ \\
\hline Khositseth et $\mathrm{a} /(40)$ & ND & 61 & $1(1)$ & $4(2)$ & $32(1)$ & $12(4)$ & $12(1)$ & $9(15)$ \\
\hline Devriendt et a/(30) & ND & 140 & ND & $6(\mathrm{ND})$ & 123 (ND) & ND & ND & $18(13)$ \\
\hline Lewin et $a^{(32)}$ & ND & 73 & $12(4)$ & $10(1)$ & $25(2)$ & 6 & 20 & $7(10)$ \\
\hline Anaclerio et $a^{(38)}$ & $1 d-5 a$ & 329 & ND (8) & ND (4) & ND (10) & ND (5) & - & $27(8)$ \\
\hline Takahashi et a/(29) & $1 m-16 a$ & 64 & $3(1)$ & 2 & $30(3)$ & - & $29(1)$ & $5(8)$ \\
\hline Ziolkowska et a/(41) & $0-20 a$ & 214 & $1(1)$ & $15(3)$ & 126 (ND) & 18 (ND) & 54 & $15(7)$ \\
\hline Beauchesne et $a /(39)$ & $18 a-70 a$ & 103 & - & $3(1)$ & $77(3)$ & $23(2)$ & - & $6(6)$ \\
\hline Lammer et al (2009) & ND & 485 & - & - & $296(30)$ & - & 189 & $30(6)$ \\
\hline Voigt et al (2002) ${ }^{(36)}$ & $4 d-58 a$ & 100 & - & $5(1)$ & $37(3)$ & - & 58 & $4(4)$ \\
\hline
\end{tabular}

*Os números entre parênteses correspondem aos pacientes com a SD22q11 e o respectivo defeito cardíaco conotruncal. N: número de pacientes: RN: recém-nascidos; d: dias; m: meses; a: anos; ND: não descrito; IAA: interrupção de arco aórtico; TA: truncus arteriosus; TOF: tetralogia de Fallot; CIVIAP: comunicação interventricular com atresia pulmonar. 
grande proporção de fetos com defeitos cardíacos e SD22q11 acaba não sendo diagnosticada durante o período pré-natal, seja por uma interpretação equivocada de malformações cardíacas maiores durante a avaliação ultrassonográfica fetal, especialmente de defeitos que envolvem as vias de saída do coração, seja pela presença de defeitos cardíacos menores, como anomalias isoladas da ramificação do arco aórtico, que são de difícil detecção pela ecocardiografia fetal ${ }^{(61)}$.

Do ponto de vista clínico, a SD22q11 é diagnosticada principalmente em indivíduos com cardiopatia congênita com achados sindrômicos e/ou associados a anormalidades extracardíacas ${ }^{(18,20,22,24,26,62,63)}$. Contudo, relatos da deleção em crianças com cardiopatia congênita isolada, apesar de pouco frequentes, também são verificados ${ }^{(28,34)}$. É possível que alguns desses indivíduos apresentem características sindrômicas não facilmente detectadas na infância ${ }^{(26,47)}$. Os achados faciais, por exemplo, caracterizam-se pela presença de um aumento do comprimento vertical da face; hipertelorismo (distância interpupilar aumentada); fendas palpebrais estreitas e oblíquas para cima; aumento da altura do nariz, com sua base e narinas pequenas e um enchimento sobre sua ponte, fazendo com que o mesmo apresente um formato tubular ou cilíndrico; redundância das pálpebras superiores (hooding); achatamento malar; retrognatia e anormalidades menores de orelhas (especialmente sobredobramento das hélices, orelhas arredondadas e baixo implantadas). Entretanto, essas alterações podem passar despercebidas, principalmente durante os primeiros anos de vida, e sofrerem influência da etnia do paciente. As mudanças fenotípicas associadas ao crescimento são bem conhecidas, sendo que a aparência facial dos pacientes com a SD22q11 tende a ficar mais distinta com a idade ${ }^{(13,26)}$ (Figura 1). Em indivíduos de mais idade, a apresentação torna-se também mais óbvia, pois muitas anormalidades, como o atraso no desenvolvimento neuropsicomotor, os distúrbios de fala, a insuficiência velofaríngea e os transtornos comportamentais, frequentes em pacientes com a SD22q11, tornam-se evidentes ${ }^{(13)}$. Isso é de grande importância, pois a maioria dos pacientes com cardiopatia congênita é usualmente avaliada por um especialista em uma idade precoce, podendo a doença, assim, passar despercebida na avaliação ${ }^{(26)}$.

Por outro lado, é preciso estar ciente de que o atraso do desenvolvimento e o déficit cognitivo observado em pacientes com a SD22q11 não são atribuídos à condição cardíaca ou ao seu tratamento. Eles representam, provavelmente, um componente da própria síndrome ${ }^{(55)}$. As anormalidades em órgãos abdominais, como malformações renais comuns na SD22q11, muitas vezes apresentam-se de forma assintomática, podendo não ser identificadas até a realização de um exame de imagem, como o ultrassom abdominal ${ }^{(26)}$. Apesar dessas dificuldades, o reconhecimento dos achados extracardíacos é de grande importância, pois os mesmos

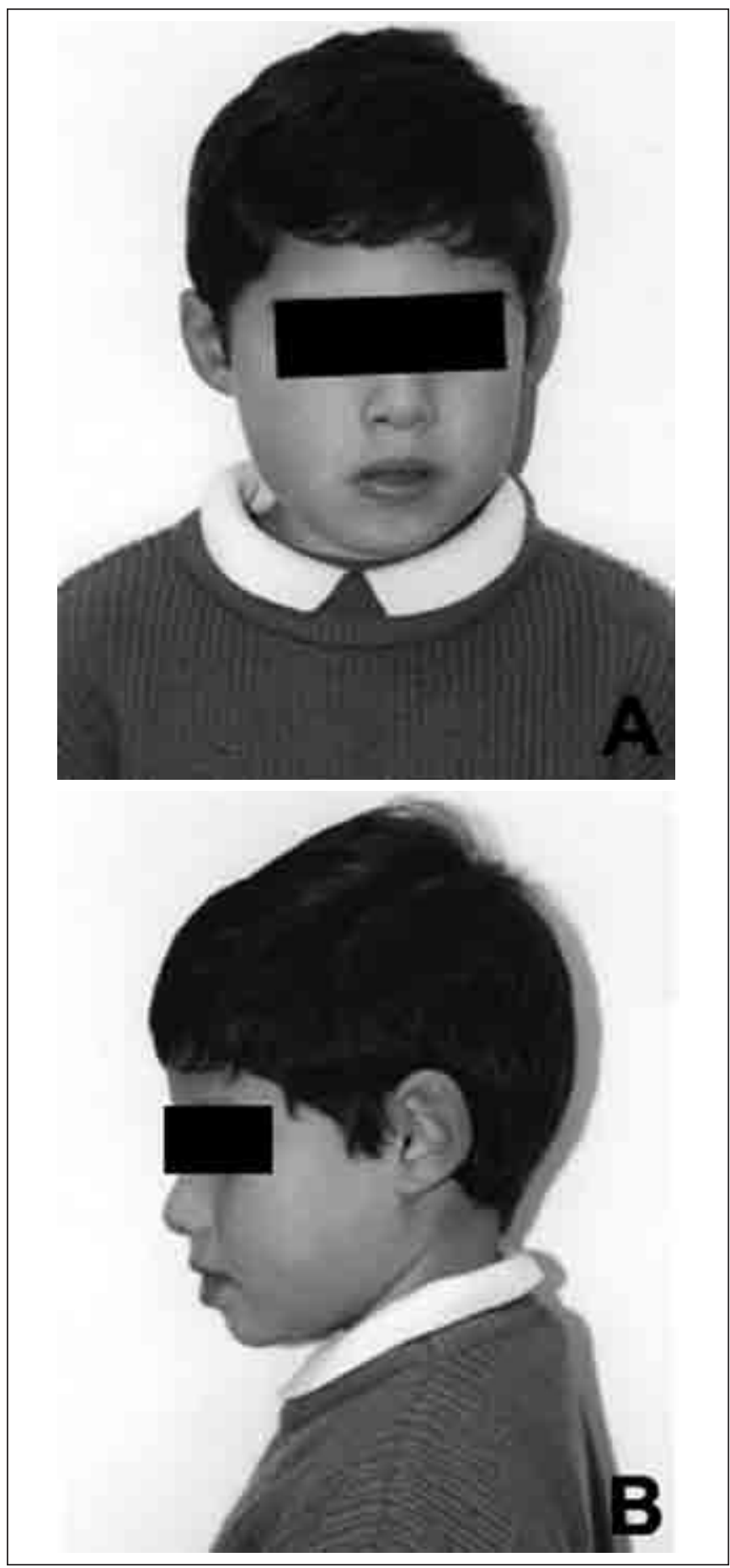

Figura 1 - Características craniofaciais de uma criança portadora da SD22q11 (visão de frente-A e perfil-B). Notar principalmente o sobredobramento da hélice da orelha esquerda $(B)$. 
podem auxiliar na suspeita clínica e no diagnóstico precoce dos pacientes com a SD22q11 ${ }^{(13)}$.

A SD22q11 tem sido pouco descrita em indivíduos com história familiar de cardiopatia congênita. É possível que esse aspecto tenha relação tanto com a grande variabilidade clínica observada entre pacientes afetados, inclusive em uma mesma família, como ao fato de que apenas uma parcela menor dos casos relatados dessa síndrome seja realmente herdada ${ }^{(13,26)}$.

\section{Triagem para SD22q11 entre portadores de cardiopatia congênita}

Numerosos estudos demonstram o grande impacto clínico da SD22q11 em pacientes portadores de cardiopatia congênita. Contudo, os mesmos têm levado muitos clínicos à falsa impressão de que qualquer paciente com malformação cardíaca deva realizar o teste de FISH para o diagnóstico. No intuito de evitar atrasos no diagnóstico, identificar outros membros da família afetados, reduzir o número de testes genéticos desnecessários e melhorar a acurácia do diagnóstico, existem diversas recomendações na literatura quanto aos pacientes que deveriam ser testados pela técnica de $\mathrm{FISH}^{(22)}$. A triagem em pacientes com anomalias cardíacas conotruncais, por exemplo, já é realizada rotineiramente em alguns centros ${ }^{(64)}$. Entretanto, essa parece limitada, pois um número significativo de pacientes com a SD22q11 apresenta malformações cardíacas que não pertencem a esse grupo de defeitos. Além disso, a síndrome tem sido pouco associada mesmo a alguns defeitos conotruncais, como a TGA e a DVSVD ${ }^{(43,56)}$, tornando essa abordagem algo dispendiosa. Atualmente, é grande a discussão sobre quais os pacientes deveriam ser submetidos à triagem. Porém, talvez com a implementação de novas técnicas moleculares com um custo inferior ao FISH, como a de PCR, os critérios clínicos de inclusão para testagem possam se tornar menos rígidos e, consequentemente, mais abrangentes e sensíveis.

\section{Importância da identificação de indivíduos com a SD22q11}

Os cardiologistas pediátricos e cirurgiões cardíacos devem estar cientes das peculiaridades e dos cuidados associados à SD22q11. Os pacientes afetados frequentemente apresentam alterações envolvendo vários sistemas e necessitam de um número elevado de intervenções clínicas e de hospitalizações ao longo de suas vidas, sendo que a cirurgia cardíaca representa seu principal motivo de internação em unidades de tratamento intensivo pediátricas (cerca de $57 \%$ dos casos) $)^{(13)}$.

Sabe-se também que os pacientes com a SD22q11 apresentam os mais altos índices de mortalidade, quando comparados a outras síndromes genéticas (como a síndrome de Down) e a casos não sindrômicos de cardiopatia congênita. A presença de anormalidades orofaríngeas, laringobrônquicas, imunológicas, endócrinas e psiquiátricas, frequentes na SD22q11, pode levar a várias complicações clínicas, desde o momento da indução anestésica até durante e após a realização dos procedimentos cirúrgicos. Além disso, a presença de malformações cardiovasculares adicionais pode dificultar a correção cirúrgica e, consequentemente, interferir no prognóstico ${ }^{(55,65)}$.

A SD22q11 é considerada um fator de risco para a morte pós-cirúrgica imediata em pacientes com CIV/AP e IAA. Esses indivíduos podem, muitas vezes, beneficiar-se de uma avaliação cardíaca por meio de ressonância nuclear magnética, com o objetivo de otimizar o diagnóstico de anormalidades vasculares adicionais com ou sem compressão brônquica, incluindo anomalias da posição e ramificação do arco aórtico, e de artérias pulmonares e sistêmicas. $\mathrm{O}$ cateterismo é considerado obrigatório em casos de CIV/AP do tipo B e $\mathrm{C}$, no intuito de definir a vasculatura pulmonar e prover o substrato para a avaliação morfométrica pré-operatória de fontes de sangue pulmonar necessárias para realizar o tratamento cirúrgico ${ }^{(55,65)}$.

Além disso, recomendam-se, nos pacientes com a SD22q11, avaliações regulares dos níveis séricos de cálcio (pois a hipocalcemia pode-se manifestar a qualquer momento ou idade), análise de populações linfocíticas antes de transfusões sanguíneas, administração de componentes do sangue irradiados e com sorologia negativa para o citomegalovírus em pacientes imunocomprometidos, bem como o tratamento agressivo de infecções perioperatórias, com profilaxia antimicrobiana e antifúngica. A instabilidade vasomotora, o broncoespasmo e o sangramento de vias aéreas são também complicações possíveis que devem receber atenção ${ }^{(65)}$. No Quadro 2 está listada uma série de anormalidades a serem consideradas no manejo anestésico e cirúrgico desses pacientes.

Assim, além da avaliação cardiológica, esses pacientes necessitam de acompanhamento multidisciplinar, envolvendo especialidades como Pediatria, Genética Médica, Cardiologia, Otorrinolaringologia, Cirurgia Plástica, Imunologia, 
Quadro 2 - Anormalidades a serem consideradas no manejo anestésico e cirúrgico de pacientes com a SD22q11

\begin{tabular}{|c|c|}
\hline \multicolumn{2}{|c|}{ Achados clínicos } \\
\hline Neurológicos & Vasculares \\
\hline Déficit cognitivo $^{\dagger}$ & Arco aórtico à direita ${ }^{\dagger}$ \\
\hline 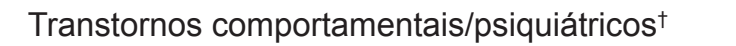 & Artéria subclávia aberrante $^{\dagger}$ \\
\hline Hipotonia generalizada ${ }^{\dagger}$ & Anel vascular \\
\hline Crises convulsivas $^{\dagger}$ & 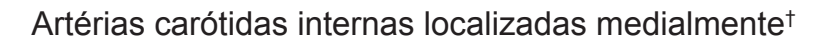 \\
\hline Otorrinolaringológicas & Carótidas internas tortuosas, ausentes ou acessórias ${ }^{\dagger}$ \\
\hline Face de choro assimétrica & Anomalias de veias jugulares \\
\hline 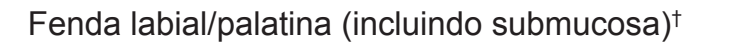 & Tortuosidade ou ausência unilateral de artéria vertebral \\
\hline Insuficiência velofaríngea (usualmente severa) ${ }^{\dagger}$ & Bifurcação baixa da artéria carótida comum \\
\hline Ausência congênita de dentes & Fenômeno de Raynaud \\
\hline Micro/retrognatia $^{\dagger}$ & Abdominais \\
\hline Sequência de Pierre Robin ${ }^{\dagger}$ & Hérnia diafragmática \\
\hline Dificuldades alimentares $^{\dagger}$ & Anomalias anais (ânus imperfurado ou mal posicionado) \\
\hline Refluxo gastroesofágico & Constipação crônica \\
\hline Hipotonia faríngea & Hematológicas/ imunológicas \\
\hline Paresia de corda vocal unilateral & Trombocitopenia/ Doença de Bernard-Soulier ${ }^{\dagger}$ \\
\hline Membrana laríngea anterior & Imunodeficiência ${ }^{\dagger}$ \\
\hline Laringomalácia & Endocrinológicas \\
\hline Hiperplasia de aritenoides & 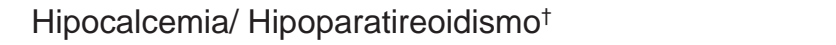 \\
\hline Traqueobroncomalácia & Hipotireoidismo \\
\hline Infecções respiratórias frequentes & Ortopédicas \\
\hline & Escoliose \\
\hline & Hemivértebras \\
\hline & Fusão de vértebras (especialmente cervicais) \\
\hline & Costelas extras ou fusionadas \\
\hline
\end{tabular}

* Baseado em Rosa et $\mathrm{al}^{(13)}{ }^{\dagger}{ }^{\dagger}$ achados mais frequentemente descritos na SD22q11.

Endocrinologia e Psiquiatria ${ }^{(13,16)}$. Contudo, como exposto, a sua identificação depende diretamente de técnicas laboratoriais de citogenética e biologia molecular que não são rotineiramente realizadas em nosso meio devido ao seu alto custo, o que leva a um baixo índice de diagnóstico. Frente à importância dessa síndrome e às implicações que seu reconhecimento traz, tanto para a avaliação como para o manejo dos pacientes e de suas famílias, há justificativa para a implantação de mais centros com capacidade de desenvolver essas técnicas e, com isso, elevar o índice de detecção dos pacientes com a SD22q11.

\section{Agradecimentos}

À Coordenação de Aperfeiçoamento de Pessoal de Nível Superior (CAPES) pela bolsa de estudos recebida.

\section{Referências bibliográficas}

1. Grech V. The evolution of diagnostic trends in congenital heart disease: a population-based study. J Paediatr Child Health 1999;35:387-91.

2. Acharya G, Sitras V, Maltau JM, Dahl LB, Kaaresen PI, Hanssen TA et al. Major congenital heart disease in Northern Norway: shortcomings of pre- and postnatal diagnosis. Acta Obstet Gynecol Scand 2004;83:1124-9.

3. Hoffman JI, Kaplan S. The incidence of congenital heart disease. J Am Coll Cardiol 2002;39:1890-900.

4. Srivastava D. Genetic assembly of the heart: implications for congenital heart disease. Annu Rev Physiol 2001;63:451-69.
5. Chew C, Stone S, Donath SM, Penny DJ. Impact of antenatal screening on the presentation of infants with congenital heart disease to a cardiology unit. J Paediatr Child Health 2006;42:704-8.

6. Kapil D, Bagga A. The profile and outcome of patients admitted to a pediatric intensive care unit. Indian J Pediatr 1993;60:5-10.

7. Begić $H$, Tahirović $H$, Mesihović-Dinarević S, Ferković V, Atić N, Latifagić A. Epidemiological and clinical aspects of congenital heart disease in children in Tuzla Canton, Bosnia-Herzegovina. Eur J Pediatr 2003;162:191-3. 
8. Goldmuntz E. The epidemiology and genetics of congenital heart disease. Clin Perinatol 2001;28:1-10.

9. Gawde H, Patel ZM, Khatkhatey MI, D'Souza A, Babu S, Adhia R et al. Chromosome 22 microdeletion by F.I.S.H. in isolated congenital heart disease. Indian J Pediatr 2006;73:885-8.

10. Johnson MC, Hing A, Wood MK, Watson MS. Chromosome abnormalities in congenital heart disease. Am J Med Genet 1997;70:292-8.

11. Stumm M, Tönnies H, Wieacker PF. Molecular cytogenetic techniques for the diagnosis of chromosomal abnormalities in childhood disease. Eur J Pediatr 1999;158:531-6.

12. Online Mendelian Inheritance in Man (OMIM) [homepage on the Internet]. Baltimore e Bethesda: BeMcKusick-Nathans Institute for Genetic Medicine, Johns Hopkins University and National Center for Biotechnology Information, National Library of Medicine [cited 2009 Dec 05]. Available from: http://www. ncbi.nlm.nih.gov/omim/

13. Rosa RF, Zen PR, Roman T, Graziadio C, Paskulin GA. 22q11.2 deletion syndrome: catching the CATCH22. Rev Paul Pediatr 2009;27:211-20.

14. Koshiyama DB, Rosa RF, Zen PR, Pereira VL, Graziadio C, Coser VM et al. Síndrome de deleção 22q11.2: importância da avaliação clínica e técnica de FISH. Rev Assoc Med Bras 2009;55:442-6.

15. Sandrin-Garcia P, Abramides DV, Martelli LR, Ramos ES, Richieri-Costa A, Passos GA. Typical phenotypic spectrum of velocardiofacial syndrome occurs independently of deletion size in chromosome 22q11.2. Mol Cell Biochem 2007;303:9-17.

16. Kapadia RK, Bassett AS. Recognizing a common genetic syndrome: 22q11.2 deletion. CMAJ 2008;178:391-3.

17. Gioli-Pereira L, Pereira AC, Mesquita SM, Lopes AA, Krieger JE. PCR screening for 22q11.2 microdeletion: development of a new cost-effective diagnostic tool. Clin Chim Acta 2006;369:78-81.

18. Fokstuen S, Arbenz U, Artan S, Dutly F, Bauresfeld U, Brecevic L et al. 22q11.2 deletions in a series of patients with non-selective congenital heart defects: incidence, type of defects and parental origin. Clin Genet 1998;53:63-9.

19. Goodship J, Cross I, LiLing J, Wren C. A population study of chromosome $22 q 11$ deletions in infancy. Arch Dis Child 1998;79:348-51.

20. Borgmann S, Luhmer I, Arslan-Kirchner M, Kallfelz HC, Schmidtke J. A search for chromosome 22q11.2 deletions in a series of 176 consecutively catheterized patients with congenital heart disease: no evidence for deletions in non-syndromic patients. Eur J Pediatr 1999;158:958-63.

21. Yong DE, Booth P, Baruni J, Massie D, Stephen G, Couzin D et al. Chromosome 22q11 microdeletion and congenital heart disease - a survey in a paediatric population. Eur J Pediatr 1999;158:566-70.

22. Marino B, Digilio MC, Toscano A, Anaclerio S, Giannotti A, Feltri C et al. Anatomic patterns of conotruncal defects associated with deletion 22q11. Genet Med 2001;3:45-8.

23. Giray Ö, Ülgenalp A, Bora E, Sagin Saylam G, Ünal N, Mese T et al. Congenital cardiac defects with 22q11 deletion. Turk J Pediatr 2003;45:217-20.

24. Botto LD, May K, Fernhoff PM, Correa A, Coleman K, Rasmussen SA et al. A population-based study of the 22q11.2 deletion: phenotype, incidence, and contribution to major birth defects in the population. Pediatrics 2003;112:101-7.

25. Schellberg R, Schwanitz G, Grävinghoff L, Kallenberg R, Trost D, Raff R et al. New trends in chromosomal investigation in children with cardiovascular malformations. Cardiol Young 2004; 14:622-9.

26. Rosa RF, Pilla CB, Pereira VL, Flores JA, Golendziner E, Koshiyama DB et al. 22q11.2 deletion syndrome in patients admitted to a cardiac pediatric intensive care unit in Brazil. Am J Med Genet 2008;146A:1655-61.

27. Hu Y, Zhu X, Yang Y, Mo X, Sheng M, Yao J et al. Incidences of microdeletion/duplication 22q11.2 detected by multiplex ligation-dependent probe amplification in patients with congenital cardiac disease who are scheduled for cardiac surgery. Cardiol Young 2009;19:179-84.

28. Goldmuntz E, Driscoll D, Budarf ML, Zackai EH, McDonald-McGinn DM, Biegel JA et al. Microdeletions of chromosomal region 22q11 in patients with congenital conotruncal cardiac defects. J Med Genet 1993;30:807-12.

29. Takahashi K, Kido S, Hoshino K, Ogawa K, Ohashi H, Fukushima Y. Frequency of a 22q11 deletion in patients with conotruncal cardiac malformations: a prospective study. Eur J Pediat 1995;154:878-81.
30. Devriendt K, Eyskens B, Swillen A, Dumoulin M, Gewillig M, Fryns JP. The incidence of a deletion in chromosome 22Q11 in sporadic and familial conotruncal heart disease. Eur J Pediatr 1996;155:721.

31. Webber SA, Hatchwell E, Barber JC, Daubeney PE, Crolla JA, Salmon AP et al. Importance of microdeletions of chromosomal region 22q11 as a cause of selected malformations of the ventricular outflow tracts and aortic arch: a three-year prospective study. J Pediatr 1996;129:26-32.

32. Lewin MB, Lindsay EA, Jurecic V, Goytia V, Towbin JA, Baldini A. A genetic etiology for interruption of the aortic arch type B. Am J Cardiol 1997;80:493-7.

33. Worthington S, Bower C, Harrop K, Loh J, Walpole I. 22q11 deletions in patients with conotruncal heart defects. J Paediatr Child Health 1998;34:438-43.

34. Goldmuntz E, Clark BJ, Mitchell LE, Jawad AF, Cuneo BF, Reed L et al. Frequency of 22q11 deletions in patients with conotruncal defects. J Am Coll Cardiol 1998;32:492-8.

35. Iserin L, de Lonlay P, Viot G, Sidi D, Kachaner J, Munnich A et al. Prevalence of the microdeletion 22q11 in newborn infants with congenital conotruncal cardiac anomalies. Eur J Pediatr 1998;157:881-4.

36. Voigt R, Maier-Weidmann M, Lange PE, Haaf T. Chromosome 10p13-14 and 22q11 deletion screening in 100 patients with isolated and syndromic conotruncal heart defects. J Med Genet 2002;39:e16.

37. Derbent M, Yilmaz Z, Baltaci V, Saygili A, Varan B, Tokel K. Chromosome 22q11.2 deletion and phenotypic features in 30 patients with conotruncal heart defects. Am J Med Genet 2003;116:129-35.

38. Anaclerio S, Di Ciommo V, Michielon G, Digilio MC, Formigari R, Picchio FM et al. Conotruncal heart defects: impact of genetic syndromes on immediate operative mortality. Ital Heart J 2004;5:624-8.

39. Beauchesne LM, Warnes CA, Connolly HM, Ammash NM, Grogan M, Jalal SM et al. Prevalence and clinical manifestations of 22q11.2 microdeletion in adults with selected conotruncal anomalies. J Am Coll Cardiol 2005;45:595-8.

40. Khositseth A, Tocharoentanaphol C, Khowsathit P, Ruangdaraganon N. Chromosome 22q11 deletions in patients with conotruncal heart defects. Pediatr Cardiol 2005;26:570-3.

41. Ziolkowska L, Kawalec W, Turska-Kmiec A, Krajewska-Walasek M, BrzezinskaRajszys G, Daszkowska J et al. Chromosome 22q11.2 microdeletion in children with conotruncal heart defects: frequency, associated cardiovascular anomalies, and outcome following cardiac surgery. Eur J Pediatr 2008;167:1135-40.

42. Lammer EJ, Chak JS, lovannisci DM, Schultz K, Osoegawa K, Yang W et al. Chromosomal abnormalities among children born with conotruncal cardiac defects. Birth Defects Res A Clin Mol Teratol 2009;85:30-5.

43. Momma K. Cardiovascular anomalies associated with chromosome 22q11.2 deletion. Int J Cardiol 2007;114:147-9.

44. Carotti A, Marino B, Di Donato RM. Influence of chromosome 22q11.2 microdeletion on surgical outcome after treatment of tetralogy of fallot with pulmonary atresia. J Thorac Cardiovasc Surg 2003;126:1666-7.

45. Rauch A, Hofbeck M, Leipold G, Klinge J, Trautmann U, Kirsch M et al. Incidence and significance of 22q11.2 hemizygosity in patients with interrupted aortic arch. Am J Med Genet 1998;78:322-31.

46. Marino B, Digilio MC, Persiani M, Di Donato R, Toscano A, Giannotti A et al. Deletion 22q11 in patients with interrupted aortic arch. Am J Cardiol 1999;84:360-1.

47. McElhinney DB, Driscoll DA, Emanuel BS, Goldmuntz E. Chromosome 22q11 deletion in patients with truncus arteriosus. Pediatr Cardiol 2003;24:569-73.

48. Mahle WT, Crisalli J, Coleman K, Campbell RM, Tam VK, Vincent RN et al. Deletion of chromosome 22q11.2 and outcome in patients with pulmonary atresia and ventricular septal defect. Ann Thorac Surg 2003;76:567-1.

49. Hofbeck M, Leipold G, Rauch A, Buheitel G, Singer H. Clinical relevance of monosomy 22q11.2 in children with pulmonary atresia and ventricular septal defect. Eur J Pediatr 1999;158:302-7.

50. Marino B, Digilio MC. Congenital heart disease and genetic syndromes: specific correlation between cardiac phenotype and genotype. Cardiovasc Pathol 2000;9:303-15.

51. Amati F, Mari A, Digilio MC, Mingarelli R, Marino B, Giannotti A et al. 22q11 deletions in isolated and syndromic patients with tetralogy of Fallot. Hum Genet 1995;95:479-82. 
52. Trainer AH, Morrison N, Dunlop A, Wilson N, Tolmie J. Chromosome 22q11 microdeletions in tetralogy of Fallot. Arch Dis Child 1996;74:62-3.

53. Maeda J, Yamagishi H, Matsuoka R, Ishihara J, Tokumura M, Fukushima H et al. Frequent association of 22q11.2 deletion with tetralogy of Fallot. Am J Med Genet 2000;92:269-72.

54. McElhinney DB, McDonald-McGinn D, Zackai EH, Goldmuntz E. Cardiovascular anomalies in patients diagnosed with a chromosome 22q11 deletion beyond 6 months of age. Pediatrics 2001;108:E104.

55. Carotti A, Digilio MC, Piacentini G, Saffirio C, Di Donato RM, Marino B. Cardiac defects and results of cardiac surgery in 22q11.2 deletion syndrome. Dev Disabil Res Rev 2008;14:35-42.

56. Repetto GM, Guzmán ML, Puga A, Calderón JF, Astete CP, Aracena M et al. Clinical features of chromosome 22q11.2 microdeletion syndrome in 208 Chilean patients. Clin Genet 2009;76:465-70.

57. Cohen E, Chow EW, Weksberg R, Bassett AS. Phenotype of adults with the 22q11 deletion syndrome: A review. Am J Med Genet 1999;86:359-65.

58. Bassett AS, Chow EW, Husted J, Weksberg R, Caluseriu O, Webb GD et al. Clinical features of 78 adults with 22q11 Deletion Syndrome. Am J Med Genet 2005;138:307-13.

59. Kyburz A, Bauersfeld U, Schinzel A, Riegel M, Hug M, Tomaske M et al. The fate of children with microdeletion 22q11.2 syndrome and congenital heart defect: clinical course and cardiac outcome. Pediatr Cardiol 2008;29:76-83.

60. Shooner KA, Rope AF, Hopkin RJ, Andelfinger GU, Benson DW. Genetic analyses in two extended families with deletion 22q11 syndrome: importance of extracardiac manifestations. J Pediatr 2005;146:382-7.

61. Volpe P, Marasini M, Caruso G, Marzullo A, Buonadonna AL, Arciprete P et al. 22q11 deletions in fetuses with malformations of the outflow tracts or interruption of the aortic arch: impact of additional ultrasound signs. Prenat Diagn 2003;23:752-7.

62. Digilio MC, Marino B, Dallapiccola B. Deletion 22q11 and isolated congenital heart disease. Int J Cardiol 2008;123:364-5.

63. Belangero SI, Bellucco FT, Kulikowski LD, Christofolini DM, Cernach MC, Melaragno MI. 22q11.2 deletion in patients with conotruncal heart defect and del22q syndrome phenotype. Arq Bras Cardiol 2009;92:307-11.

64. Butts SC, Tatum SA 3rd, Mortelliti AJ, Shprintzen RJ. Velo-cardio-facial syndrome: the pediatric otolaryngologist's perspective. Curr Opin Otolaryngol Head Neck Surg 2005;13:371-5.

65. Formigari R, Michielon G, Digilio MC, Piacentini G, Carotti A, Giardini A et al Genetic syndromes and congenital heart defects: how is surgical management affected? Eur J Cardiothorac Surg 2009;35:606-14 